\title{
Computational Mechanistic Study of Thionation of Carbonyl Compounds with Lawesson's Reagent
}

\author{
L. Legnani, ${ }^{\dagger}{ }^{\dagger}$ L. Toma, $^{\dagger}$ P. Caramella, ${ }^{\dagger}$ M. A. Chiacchio, ${ }^{\dagger}+$ S. Giofrè, ${ }^{\S}$ I. Delso, ${ }^{\|}$T. Tejero, ${ }^{\|}$ \\ and P. Merino*, \\ ${ }^{\dagger}$ Dipartimento di Chimica, Università di Pavia, Via Taramelli 12, 27100 Pavia, Italy \\ ${ }^{\ddagger}$ Dipartimento di Scienze del Farmaco, Università di Catania, V. le A. Doria 6, 95125 Catania, Italy \\ ${ }^{\S}$ Dipartimento di Scienze chimiche, biologiche, farmaceutiche e ambientali, Università di Messina, Via Stagno d'Alcontres, 98166 \\ Messina, Italy \\ "Laboratorio de Síntesis Asimétrica, Instituto de Síntesis Quimica y Catalisis Homogenea (ISQCH), Universidad de Zaragoza-CSIC, \\ Zaragoza 50009, Spain
}

\section{Supporting Information}

ABSTRACT: The thionation reaction of carbonyl compounds with Lawesson's reagent (LR) has been studied using density functional theory methods and topological analyses. After dissociation of LR, the reaction takes place through a twostep mechanism involving (i) a concerted cycloaddition between one monomer and the carbonyl compound to form a four-membered intermediate and (ii) a cycloreversion leading to the thiocarbonyl derivative and phenyl(thioxo)phosphine oxide. Topological analyses confirmed the concertedness and asynchronicity of the process. The second step is the rate-limiting one, and the whole process resembles the currently accepted mechanism for the lithium salt-free Wittig reaction. No zwitterionic intermediates are formed during the reaction, although stabilizing electrostatic interactions are present in initial stages. Phenyl(thioxo)phosphine oxide formed in the thionation reaction is capable of performing a second thionation, although with energy barriers higher than the first one. The driving force of the

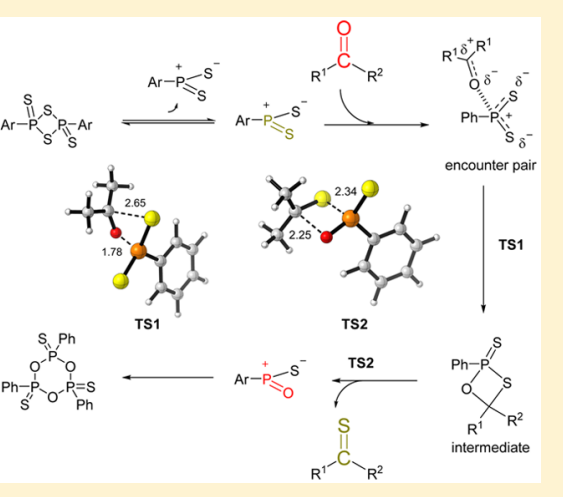
thionation reactions is the formation of trimers from the resulting monomers. In agreement with experimental observations, the amides are the most reactive when compared with esters, aldehydes, and ketones and the reaction is slightly influenced by the polarity of the solvent. Whereas for amides and esters substituents have little effect, aldehydes and ketones are influenced by both steric and electronic effects.

\section{INTRODUCTION}

The thiocarbonyl group is widely found in a great variety of organic compounds. ${ }^{1}$ Thio analogues of ketones, lactones, amides, and esters are very important biological molecules, and they are widely used in medicine as therapeutic agents with a wide range of biological activities. ${ }^{2}$ Thiocarbonyl compounds have also been widely used in organic synthesis as precursors of organosulfur compounds ${ }^{3}$ and can participate in essentially the same reactions of the counterpart carbonyl derivatives, although showing, in general, a lower reactivity. Furthermore, thiocarbonyl compounds have a rich photochemistry providing excited states that lead to various photoproducts. ${ }^{4}$ The more expeditious way of preparing a thiocarbonyl derivative is the direct thionation of the corresponding carbonyl compound. ${ }^{5}$ This transformation can be performed with a variety of reagents, including phosphorus pentasulfide, ${ }^{6}$ hydrogen sulfide, ${ }^{7}$ thiophosphoryl chloride, ${ }^{8}$ bis(trimethylsilyl) sulfide, ${ }^{9}$ and rhodanine. $^{10}$ In 1956, Lecher and co-workers introduced reagent $1,{ }^{11}$ known as Lawesson's reagent [LR (Figure 1)], ${ }^{12}$ because of further extensive investigations of Lawesson and coworkers. ${ }^{13}$ Compound $\mathbf{1}$ performs the direct conversion of

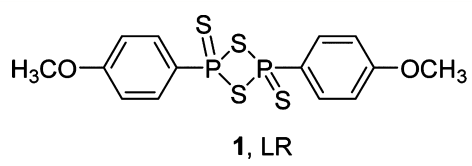

Figure 1. 2,4-Bis( $p$-methoxyphenyl)-1,3-dithiadiphosphetane-2,4-disulfide (Lawesson's reagent).

ketones, aldehydes, lactones, esters, amides, and carboxylic acids into the corresponding thiocarbonyl derivatives in high yields with easy handling. ${ }^{14}$ Since then, there has been a huge volume of work on thionation reactions of carbonyl compounds using Lawesson's reagent. ${ }^{15}$ The diphenyl-1,3-dithiadiphosphetane-2,4-disulfide has been also used with similar results, ${ }^{16}$ but the lack of commercial availability prevented its wider application.

The reactivity of LR is not very high. Nishio et al. reported that hydroxyl groups were the most reactive ones followed in order by amides, ketones, and esters. ${ }^{17}$ In general, typical

Received: June 13, 2016

Published: July 26, 2016 
conditions require an excess of LR, and the reaction is conducted under dry conditions at elevated temperatures in hydrocarbon solvents (toluene, xylene, and benzene), affording moderate yields in slow reactions $(2-25 \mathrm{~h})$ that often produce some undesired byproduct. The use of microwaves accelerates considerably the reaction, ${ }^{8,18}$ particularly when it is conducted under solvent-free conditions. ${ }^{19}$ In this way, reactions become cleaner and faster and are easy to manipulate. Moreover, solvent-free conditions provide the opportunity to work in open vessels, preventing the risk of development of high pressure.

Despite the utility of the thionation reaction using LR and the different reactivity observed depending on the thiocarbonyl derivative, the only reports regarding mechanistic investigations of the thionation of carbonyls are those of Rauchfuss ${ }^{20}$ and Yoshifuji ${ }^{21}$ in 1986 and 1994, respectively. Some mechanistic proposals have been made for thionation of carboxylic acids ${ }^{22}$ and alcohols ${ }^{23}$ but without any experimental or computational support. In fact, the study of these derivatives requires a more elaborate approach because different mechanisms can operate as in the case of $N$-alkylhydroxamic acids. ${ }^{24}$ The mechanism of thionation of a carbonyl group is globally accepted to consist of the cycloaddition of a reactive dithiophosphine ylide 2 (in equilibrium Lawesson's reagent 1 ) to the carbonyl derivative 3 and to yield a thiaoxaphosphetane intermediate 4 , which further evolves to thiocarbonyl group $\mathbf{5}$ and an oxathiophosphine ylide 6 through a cycloreversion reaction (Scheme 1). The two steps

Scheme 1. Accepted Mechanism of Thionation of Carbonyls by Lawesson's Reagent

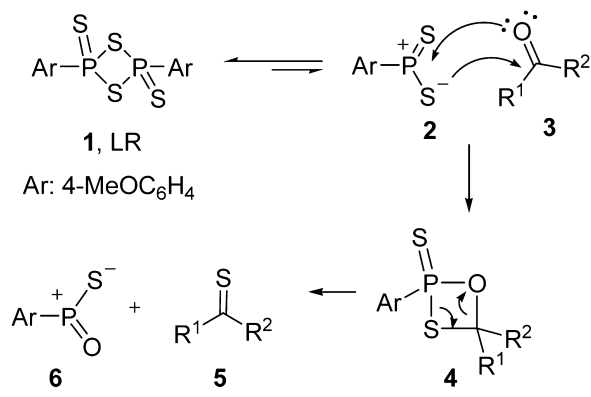

closely resemble those of the Wittig reaction. ${ }^{25}$ Until now, the driving force of the Lawesson reaction was considered the formation of the most stable $\mathrm{P}=\mathrm{O}$ bond in byproduct $\mathbf{6}^{26}$

However, although previous experimental work supports this mechanism, ${ }^{20,21}$ no information about the nature of the transition states involved in the reaction and the different reactivities observed between diverse carbonyl derivatives exists. Herein, we report the first systematic computational study of the thionation of several carbonyl functionalities, including aldehydes, ketones, esters, and amides, with Lawesson's reagent. ${ }^{27}$ The molecular mechanism of the reaction, the different reactivities observed for carbonyl derivatives, and the nature of the transition states will be discussed.

\section{COMPUTATIONAL METHODS}

All of the calculations were performed using Gaussian09. ${ }^{28}$ Computations were conducted using Truhlar's M06-2X functional. ${ }^{29}$ Standard basis sets $6-31 \mathrm{G}(\mathrm{d})$ and $6-311 \mathrm{G}(\mathrm{d}, \mathrm{p})^{30}$ were employed, and diffuse functions were added in all cases. Geometry full optimizations were performed at the M06-2X/6-31G+(d,p)/PCM=DCM level, and then single-point calculations at the M06-2X/6-311+G(d,p)/ $\mathrm{PCM}=\mathrm{DCM}$ level were performed to obtain more accurate energies.
These levels of theory have provided satisfactory agreement with experimental data. ${ }^{31}$ Nevertheless, for the purpose of comparison, we have conducted single-point calculations at the SCS-MP2/6-311+G$(\mathrm{d}, \mathrm{p})$ level of theory in the case of thionation of acetone, and similar differences were observed, thus confirming the validity of the DFT methods utilized (for other details, see the Supporting Information). All discussions are based on values of free energies $(G)$. However, several of the individual reactions involved in the study are bimolecular processes. To avoid errors due to entropic effects upon comparison of all stationary points in an only energy diagram, we used corrected free energy $\left(G_{\text {corr }}\right)$ values following Sakaki's model. ${ }^{32}$ Translational and rotational degrees of freedom in solution are strongly suppressed because of the interactions with solvent molecules, and these interactions are not well-estimated by continuum solvent models like PCM; as a consequence, thermodynamic corrections to potential energies calculated by using continuum solvation models overestimate the contributions of translational and rotational degrees of freedom to the entropy. ${ }^{33}$ According to Sakaki's model, only vibrational contributions to entropy must be considered calculating free energy as illustrated in eq 1 :

$$
\Delta G_{\text {corr }}=\Delta H-T \Delta S_{\text {vib }}
$$

It has been demonstrated that $\Delta G_{\text {corr }}$ is closer to the experimentally derived $\Delta G$ that the uncorrected calculated free energy. ${ }^{34}$ We used as a model reaction thionation of acetone in DCM, but extensions to various solvents were included. For the LR, we chose the phenyl derivative hereafter indicated by LRD1. The model was further validated with benzophenone, aldehydes, esters, and amides. Formyl, methyl, and phenyl derivatives were also considered in all cases. To facilitate discussion, we named the compounds using uppercase letters according to their two initial letters (KE for ketones, AL for aldehydes, ES for esters, and $\mathbf{A M}$ for amides) followed by $\mathbf{O}$ and $\mathbf{S}$ for carbonyl and thiocarbonyl derivatives, respectively; lowercase letters indicate the substituents: for ketones, $\mathbf{a}$ and $\mathbf{b}$ refer to acetone and benzophenone, respectively, and for the rest, a refers to Me whereas $\mathbf{b}$ and $\mathbf{c}$ refer to phenyl and hydrogen groups, respectively. For intermediates, the name of the intermediate will be followed by the two initial letters to identify the functionality and lowercase letters according to the substituents. Thus, the model study was conducted with acetone KEOa to give thioacetone KESa (for a graphical explanation, see the Supporting Information).

\section{RESULTS AND DISCUSSION}

Thionation of Acetone. The first event in the thionation reaction of acetone is the reversible dissociation of $\mathbf{1}$ into reactive species PhPSS, ${ }^{17}$ which form an encounter pair ${ }^{35}$ EPLR stabilized by electrostatic interactions between $\mathrm{P}$ and $\mathrm{S}$ atoms. ${ }^{36}$ The process is endergonic by $18.0 \mathrm{kcal} / \mathrm{mol}$ and takes place through TS-LR with a barrier of 24.5 (23.8 in G) kcal/ mol (Figure 2). This value is in excellent agreement with that reported for the breaking of the $\mathrm{P}-\mathrm{S}$ bond in the $\mathrm{P}_{2} \mathrm{~S}_{2}$ ring of Davy's reagent $\{2,4$-bis(methylthio)-2,4-dithioxo-1,3,2,4-dithiadiphosphetane $\left.\left[\left(\mathrm{CH}_{3} \mathrm{~S}\right)_{2} \mathrm{P}_{2} \mathrm{~S}_{4}\right]\right\}$ estimated to be between 23.3 and $28.8 \mathrm{kcal} / \mathrm{mol}$ using the $\operatorname{CCSD}(\mathrm{T}) /$ aug-cc-pVTZ level of theory. ${ }^{37}$ Cleavage of the encounter pair EP-LR into isolated monomeric species PhPSS results in a destabilization; ${ }^{38}$ consequently, it is expected that acetone will interact with EP-LR by forming encounter pair EP01-KEa with a stabilization of $13.3 \mathrm{kcal} / \mathrm{mol}^{39}$ This encounter pair, showing a high dipolar moment $(\mu=10 \mathrm{D})$, cannot be considered as a zwitterionic intermediate species as suggested previously ${ }^{15 \mathrm{~d}}$ but a typical Lewis acid-base pair in which the oxygen atom donates an electron pair to the electron-deficient phosphorus atom giving rise to an electrostatic interaction rather to a covalent bond. This situation is confirmed by topological $\mathrm{NCI}^{40}$ and $\mathrm{ELF}^{41}$ analyses, which show the absence of a covalent bond but the presence of the electrostatic interaction 


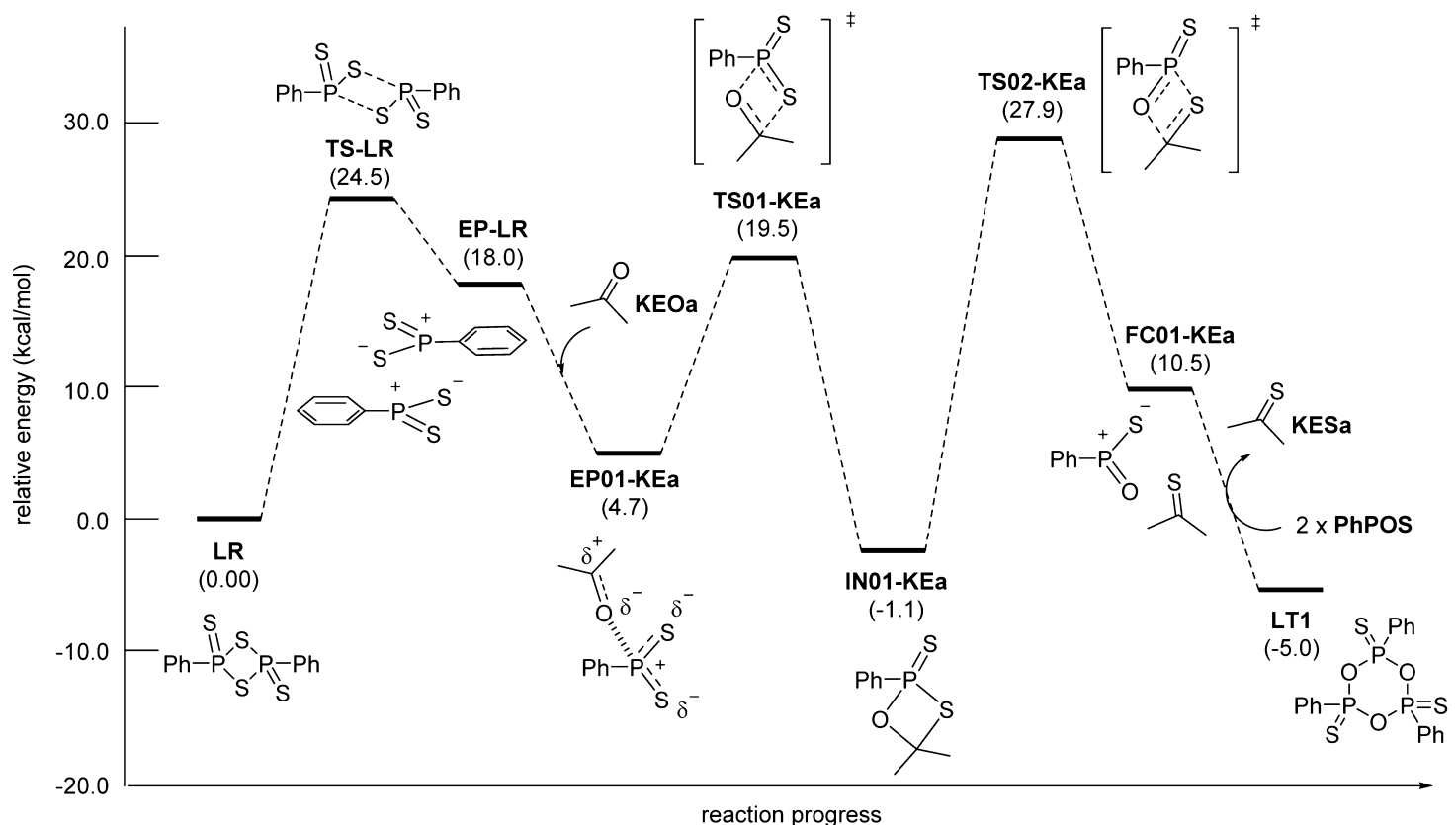

Figure 2. Energy diagram of thionation of acetone starting from Lawesson's reagent. Relative corrected free energies $\left(\Delta G_{\text {corr }}\right)$ are given in kilocalories per mole.

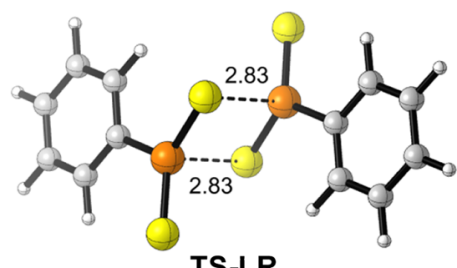

TS-LR

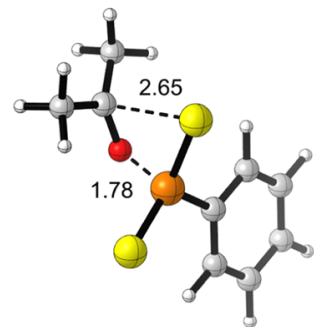

TS01-KEa

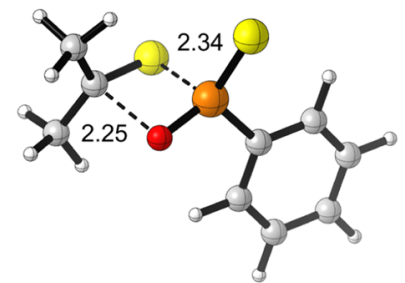

TS02-KEa

Figure 3. Optimized geometries of transition structures corresponding to the first thionation of acetone. Distances are given in angstroms. mentioned above (see the Supporting Information). Thus, at this point $[d(\mathrm{P}-\mathrm{O})=1.91 \AA]$, the $\mathrm{P}-\mathrm{O}$ bond is not yet formed. Encounter pair EP01-KEa is located $4.7 \mathrm{kcal} / \mathrm{mol}$ above LRD1 and evolves toward intermediate IN01-KEa through a first transition structure TS01-KEa in which both P$\mathrm{O}$ and $\mathrm{C}-\mathrm{S}$ bonds are being formed in a concerted albeit asynchronous way. Indeed, as indicated by ELF analysis, the reaction is highly asynchronous, the $\mathrm{P}-\mathrm{O}$ bond being formed just at the transition state, before the $\mathrm{C}-\mathrm{S}$ bond, which is formed afterward. Once intermediate IN01-KEa is formed, cycloreversion toward thioacetone KESa also takes place in a concerted asynchronous way through TS02-KEa. The cycloreversion for the second step of the reaction had been proposed previously from the first mechanistic studies. ${ }^{20}$ In this case, while the $\mathrm{C}-\mathrm{O}$ bond is broken before the transition state, the $\mathrm{P}-\mathrm{S}$ bond is broken afterward. In fact, the second transition state, TS02-KEa, is less asynchronous than the first, TS01-KEa, presumably because the $\mathrm{P}-\mathrm{O}$ interaction is stronger than the $\mathrm{P}-\mathrm{S}$ interaction developing at the end of the reaction. As expected, no electrostatic interaction between the sulfur and phosphorus atoms is found in final complex FC01-KEa formed by thioacetone KESa and byproduct PhPOS. NCI analysis confirms the presence of only a weaker van der Waals interaction between the thiocarbonyl group and the POS moiety (see the Supporting Information). Absolute energies are collected in the Supporting Information, and relative energies are given in the energy diagram of the reaction, illustrated in Figure 2. The optimized geometries of transition structures are given in Figure 3.

Scheme 2. Driving Force for the Thionation of Acetone

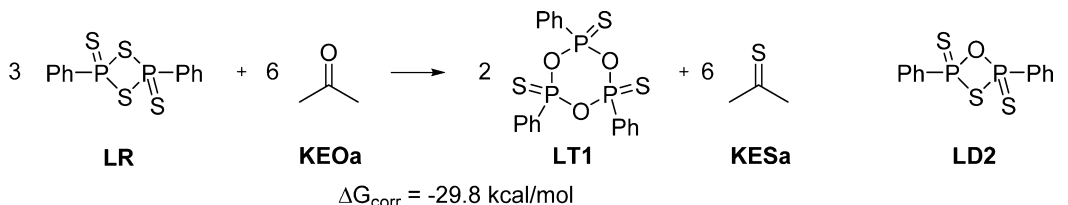




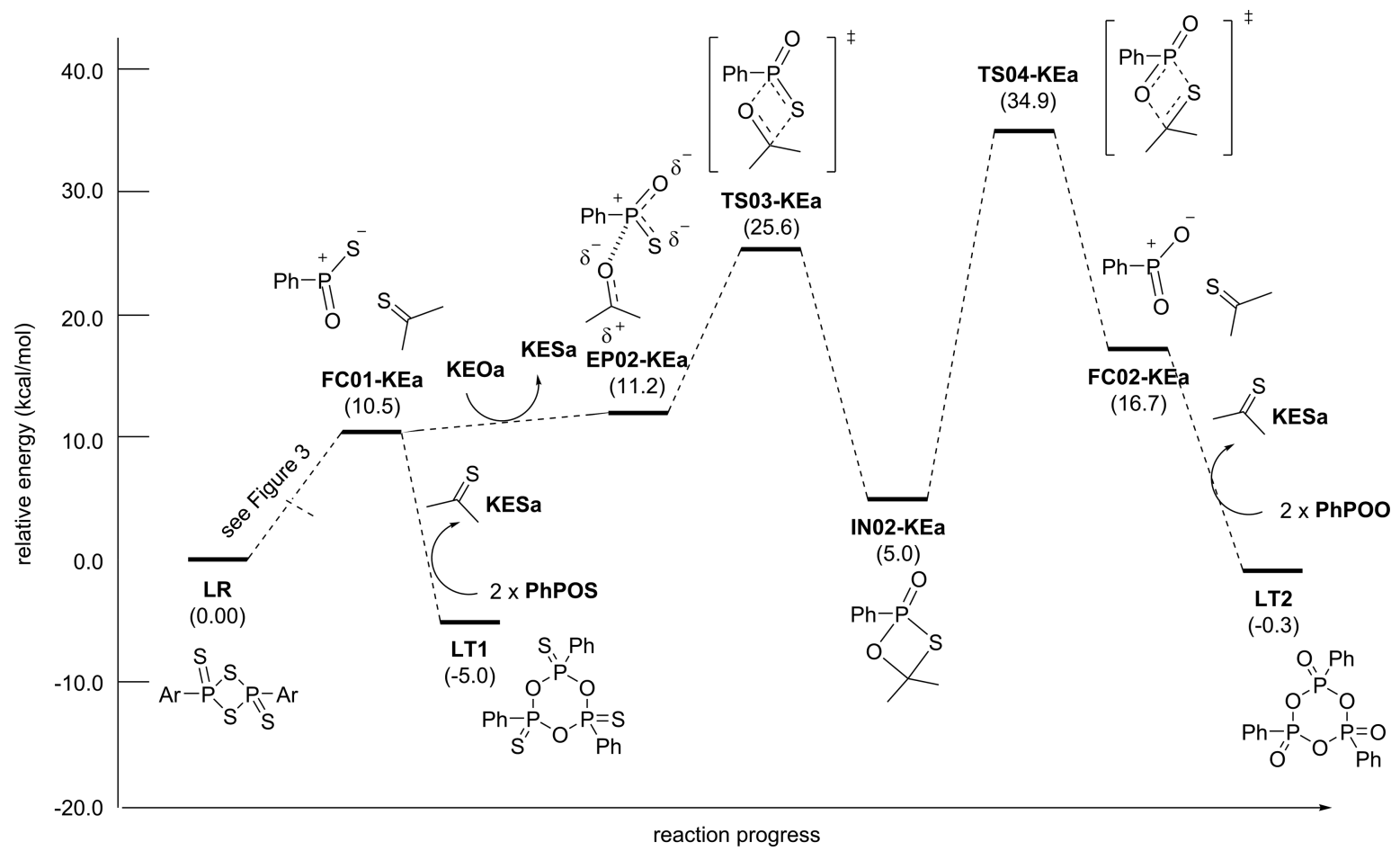

Figure 4. Energy diagram of the second thionation of acetone by PhPPOS. Relative corrected free energies $\left(\Delta G_{\text {corr }}\right)$ are given in kilocalories per mole.

Scheme 3. Thionation of Aldehydes, Esters, and Amides

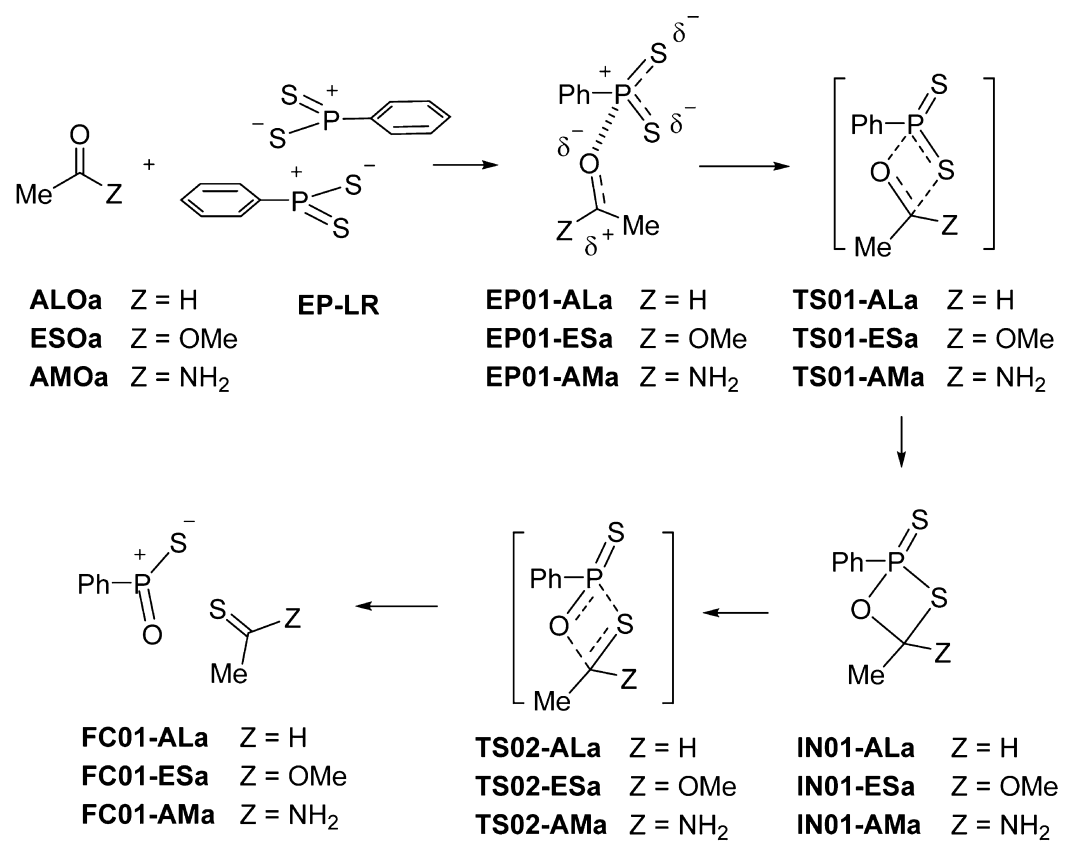

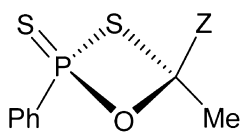

syn-IN01

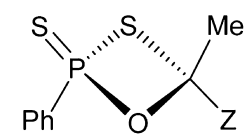

anti-IN01
The transition structure corresponding to the dissociation of Lawesson's reagent is symmetrical according to a totally concerted process, with breaking bond distances of $2.83 \AA$.
The forming bonds in TS01-KEa and TS02-KEa reveal the asynchronicity of each subprocess. As mentioned, above TS01$\mathrm{KE}$ is highly asynchronous with forming bond distances of 1.78 


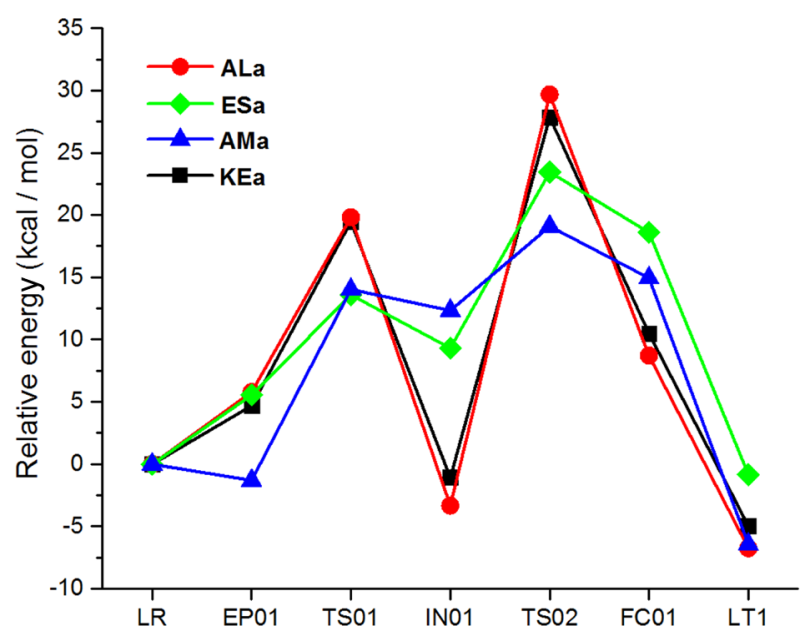

Figure 5. Comparative energy diagrams for acetone KEa, acetaldehyde ALa, methyl acetate ESa, and acetamide AMa. Relative free energies are given in kilocalories per mole.

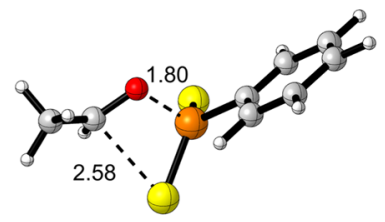

TS01-ALa-syn

(19.8)

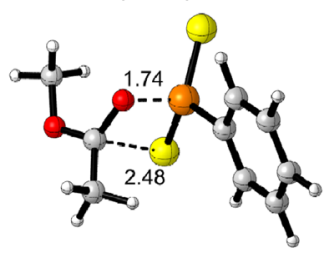

TS01-ESa-syn

(13.6)

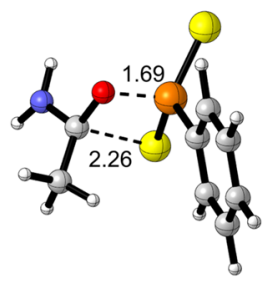

TS01-AMa-syn

(14.0)

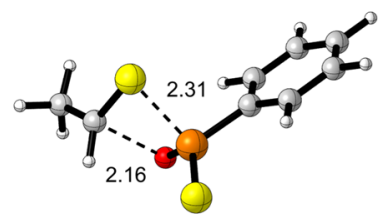

TS02-ALa-syn

(29.7)

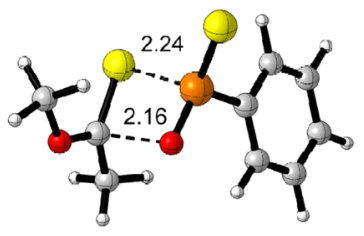

TS02-ESa-syn

(23.5)

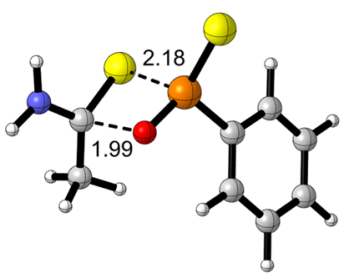

TS02-AMa-syn

(19.1)
Figure 6. Optimized geometries of transition structures corresponding to the first thionation of acetaldehyde ALOa, methyl acetate ESOa, and acetamide AMOa. Distances are given in angstroms. Relative energies are given in kilocalories per mole and are referred to Lawesson's reagent.

and $2.65 \AA$ for $\mathrm{P}-\mathrm{O}$ and $\mathrm{C}-\mathrm{S}$ bonds, respectively. On the other hand, the more synchronous TS02-KEa presents breaking bond distances of 2.25 and 2.34 for $\mathrm{C}-\mathrm{O}$ and $\mathrm{P}-\mathrm{S}$ bonds, respectively.

The rate-limiting step of acetone thionation with PhPSS corresponds to the cycloreversion process (TS02-KEa), with a barrier of $27.9 \mathrm{kcal} / \mathrm{mol}$, in agreement with that suggested by Rauchfuss on the basis of structural, spectroscopic, and kinetic studies. $^{20}$ The reaction resembles the currently accepted mechanism for the $\mathrm{Li}$ salt-free Wittig reaction ${ }^{42}$ in which an initial four-centered transition structure gives rise to a fourmembered cyclic intermediate that suffers a cycloreversion to give the products of the reaction with no ionic intermediates or polar transition states. ${ }^{43}$ Some differences, however, arise upon comparison of energetics of both processes. Whereas in the $\mathrm{Li}$ salt-free Wittig reaction the rate-determining step is the first one (cycloaddition), in the thionation reaction with Lawesson's reagent the rate-limiting step is the cycloreversion (second transition state). According to these data, in the thionation reaction, intermediate IN01-KEa should accumulate during the reaction and might be isolable under certain conditions.

The conversion of encounter pair EP01-KEa to final complex FC01-KEa is however endoergonic (by $5.8 \mathrm{kcal} /$ $\mathrm{mol}$ ), and the global reaction between LR and KEOa to give the final complex is even more endoergonic by $10.5 \mathrm{kcal} / \mathrm{mol}$; therefore, it might be considered that it is not thermodynamically favored. Some other events must compensate for this thermodynamic disfavor, providing the driving force for the thionation reaction.

We have initially searched for the formation of the crossed dimers deriving from the four possible combinations of monomer PhPOS, which is the product of the thionation reaction, and starting dithioxophosphorane PhPSS and the six combinations of monomer PhPSO. All these dimers are less stable than Lawesson dimer $\mathbf{1}$ and do not provide a significant driving force for the thionation reaction. Anticrossed dimer LD2 (Scheme 2) is however only $0.9 \mathrm{kcal} / \mathrm{mol}$ higher than 1 . Isolation of an oxothiodiphosphetane of type LD2 has indeed been reported ${ }^{21}$ with a sterically demanding aryl substituent (2,4,6-tri-isopropylphenyl), and its structure has been determined by X-ray crystallography. ${ }^{44}$ In the thionation of carbonyl compounds, trimer LT1 have been isolated ${ }^{24,45}$ and its structure has been confirmed by X-ray crystallography. ${ }^{46}$ Consequently, it is necessary to consider the overall process starting from Lawesson's reagent and acetone to give thioacetone KESa and the corresponding trimer LT1 (Scheme 2 ). According to the adjusted process illustrated in Scheme 2, the reaction has a driving force $\Delta G$ of $-5.0 \mathrm{kcal} / \mathrm{mol}$ per molecule of acetone, in agreement with the progress of the reaction. Thus, monomer PhPOS obtained as a byproduct of the thionation reaction is expected to form the trimer, pushing the reaction forward.

Byproduct PhPOS might also act as a thionating agent in a manner similar to that of PhPSS. In fact, a second thionation can occur if thioacetone is displaced in FC01-KEa by acetone, giving rise to encounter pair EP02-KEa (Figure 4).

Once EP02-KEa is formed, the reaction proceeds in a similar way to the first thionation through intermediate IN02-KEa and transition structures TS03-KEa and TS04-KEa. Again, the cycloreversion (TS04-KEa) is higher in energy than the cycloaddition (TS03-KEa), and with respect to the first thionation, the second is energetically more demanding. The driving force for the second thionation is the formation of corresponding trimer LT2. In this case, a value of only -0.3 $\mathrm{kcal} / \mathrm{mol}$ is obtained. In fact, whereas trimer LT1 has been experimentally isolated and characterized (see above), there are no reports concerning detection of trimer LT2. However, in the presence of traces of water, $\mathrm{PhPOO}$ could lead to the stable phenylphosphonic acid.

We also studied the influence of the solvent in the reaction. Thionation of acetone was calculated in the gas phase, with toluene and acetonitrile in addition to dichloromethane. In 

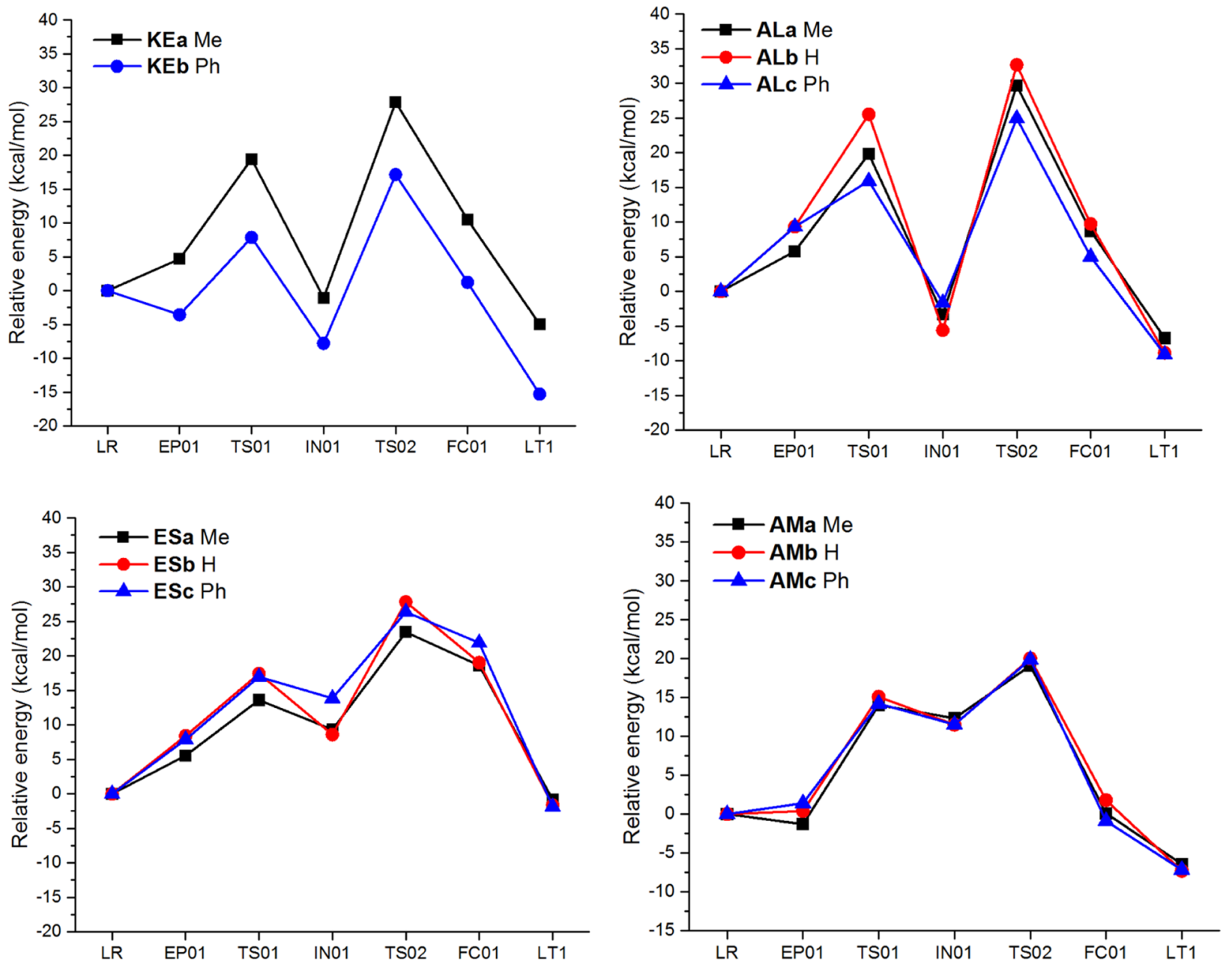

Figure 7. Comparative energy diagrams for the thionation of ketones KEOa and KEOb (top left), aldehydes ALa-c (top right), esters ESa-c (bottom left), and amides AMa-c (bottom right). Relative free energies are given in kilocalories per mole.

agreement with a concerted nonpolar reaction and the absence of zwitterionic intermediates, no significant differences were found between the different solvents studied. Nevertheless, a slight influence on the stability of encounter pairs of the carbonyl-thionating monomer was observed (for details, see the Supporting Information). This observation supports the presence of an electrostatic interaction in EP01-KEa mentioned above.

Thionation of Aldehydes, Esters, and Amides. To evaluate the thionation of other carbonyl derivatives, we expanded the study to aldehydes, esters, and amides. Acetaldehyde ALOa, methyl acetate ESOa, and acetamide AMOa were employed as the corresponding models (Scheme 3 ). In these cases, two channels are possible depending on the relative orientation of the intermediates and transition structures of the groups flanking the carbonyl and phosphorus substituents. It is noteworthy that the larger resonance effects in carbonyl compounds with respect to the thiocarbonyl ones account for the larger barrier of TS02.

We define the channel syn as that in which phosphorusphenyl and carbon-methyl substituents are on the same side of the thiaoxaphosphetane ring and the anti channel as the opposite (Scheme 3). In most cases, the syn channel was shown to be the energetically favored path by very small differences. We will base our discussion, unless otherwise mentioned, on the results obtained with syn derivatives.
The reaction with acetaldehyde, methyl acetate, and acetamide proceeds in a manner similar to that of acetone. Energetically, the less favored reaction corresponds to thionation of acetaldehyde with a barrier for the rate-limiting step (TS02) of $29.7 \mathrm{kcal} / \mathrm{mol}$, close to that of acetone $(27.9$ $\mathrm{kcal} / \mathrm{mol})$. A lower barrier is observed for methyl acetate $(24.5$ $\mathrm{kcal} / \mathrm{mol}$ ), and the lowest value corresponds to acetamide (19.1 $\mathrm{kcal} / \mathrm{mol})$. Figure 5 illustrates a comparative of the energy diagrams corresponding to the first thionation by PhPSS. It is noteworthy that the stability of IN01 (amides < esters < ketones < aldehydes) closely corresponds to the loss of resonance of carbonyl compounds in forming four-membered ring IN01. On the other hand, when we move to TS01 and TS02 the developing resonance in the products is reestablished, and consequently, the stability order of both TSs is reversed with respect to that of IN01.

According to the TS02 barriers, the predicted thionation reactivity order with Lawesson's reagent is acetaldehyde < acetone $<$ methyl acetate $<$ acetamide, in agreement with experimental results that reported the amides as the most reactive. ${ }^{17}$ In fact, it is possible to thionate an amide in the presence of an ester by conducting the reaction at ambient temperature. ${ }^{47}$ In the case of acetone and methyl acetate, the computational provision is not in agreement with experimental results. Indeed, ketones are reported to be more reactive than esters; however, thionation of the latter is greatly influenced by 
substituents, ${ }^{48}$ so it is difficult to establish a prediction of general applicability. Thionation of amides can also be influenced by steric hindrance. ${ }^{49}$ The second thionation by PhPOS has also been studied, and the same trend is observed (see the Supporting Information). Optimized geometries of transition structures TS01 and TS02 for thionation of acetaldehyde, methyl acetate, and acetamide are given in Figure 6.

As for acetone, the most asynchronous transition structure is in all cases TS01, the shortest distances corresponding to amide TS01-AMa-syn. The second transition structures are also similar to that of acetone, and again, the shortest distances correspond to the most reactive acetamide.

Effects of Substituents. In addition to the methyl group, we studied the effect of hydrogen and phenyl groups as substituents. Figure 7 illustrates comparative energy diagrams for ketones, aldehydes, esters, and amides. Only the first thionation is reported. Similar results are observed for the secod thionation (see the Supporting Information).

The thionation of aldehydes clearly shows the conjugation effects of $\mathrm{Ph}$ and $\mathrm{Me}$. The loss of conjugation influences the order of stability of IN01 $(\mathrm{Ph}<\mathrm{Me}<\mathrm{H})$, while the increase in the level of conjugation in TS01 and TS02 reverses the TS stability order $(\mathrm{Ph}>\mathrm{Me}>\mathrm{H})$. In the case of amides and esters, substituents have only a modest effect.

The case of ketones is more complicated, and for benzophenone TS01, IN01 and TS02 are significantly lower in energy than for acetone. In the case of benzophenone, the two phenyl groups, because of repulsive steric effects, do not attain coplanarity with $\mathrm{C}=\mathrm{O}$ and are twisted in a conrototary fashion to relieve $\mathrm{H} / \mathrm{H}$ repulsions. Therefore, the benzophenone geometry shows a compromise between steric and conjugation effects. In IN01, conjugation is lost but the steric hindrance between the two phenyls is also relieved. The relief of steric hindrance is dominating and leads to the stability of IN01 of benzophenone being greater than that of acetone.

\section{CONCLUSIONS}

The thionation of organic carbonyls had been rationalized as a nucleophilic attack of carbonyl oxygen upon ArPSS monomers. $^{20}$ In fact, in the initial stages of the reaction, an interaction between the carbonyl oxygen and the phosphorus atom takes place. However, this interaction has an electrostatic character and does not involve creation of any covalent bond. Consequently, the reaction does not start with a nucleophilic attack. On the contrary, the thionation of organic carbonyls with Lawesson's reagent takes place in two steps, the first being a concerted cycloaddition to give a cyclic intermediate. The second step (the rate-determining one) consists of a concerted cycloreversion in which the thiocarbonyl is formed. The different reactivity observed between diverse carbonyl groups, including aldehydes, ketones, esters, and amides, is correctly predicted. The effect of the substituents can be rationalized mainly on the basis of electronic effects for aldehydes, although no differences are observed for amides and esters. In the particular case of acetone and benzophenone, steric effects also play an important role. As expected for a concerted reaction, the solvent has little effect, and similar results are observed with toluene, dichloromethane, and acetonitrile. In summary, the thionation of carbonyl compounds with Lawesson's reagent takes place through a two-step mechanism resembling the Wittig reaction, in which the cycloreversion (second) step is the rate-limiting one.

\section{ASSOCIATED CONTENT}

\section{Supporting Information}

The Supporting Information is available free of charge on the ACS Publications website at DOI: 10.1021/acs.joc.6b01420.

Full computational methods, details of NCI and ELF calculations, details of calculations corresponding to formation of dimers, the second thionation of acetone, the effect of the solvent, the influence of the carbonyl group and substituents on the second thionation, energy data and energy diagrams for syn and anti paths, optimized geometries of transition structures, and Cartesian coordinates of optimized structures (PDF)

\section{AUTHOR INFORMATION}

\section{Corresponding Authors}

*E-mail: pmerino@unizar.es.

*E-mail: laura.legnani@unipv.it.

\section{Notes}

The authors declare no competing financial interest.

\section{ACKNOWLEDGMENTS}

This work was supported by the Spanish Ministerio de Economía y Competitividad (MINECO) (Project CTQ201344367-C2-1-P), by the Fondos Europeos para el Desarrollo Regional (FEDER), and by the Gobierno de Aragón (Zaragoza, Spain, Bioorganic Chemistry Group, E-10). The authors acknowledge the Institute of Biocomputation and Physics of Complex Systems (BIFI) at the University of Zaragoza for computer time at clusters Terminus and Memento. M.A.C. thanks the University of Catania for partial financial support.

\section{REFERENCES}

(1) (a) Schaumann, E. In Double-Bonded Functional Groups (1989); John Wiley \& Sons, Inc.: New York, 2010; pp 1269-1367. (b) Molina, M. T.; Yáñez, M.; Mó, O.; Notario, R.; Abboud, J. L. M. In PATAI'S Chemistry of Functional Groups; John Wiley \& Sons, Ltd.: New York, 2009.

(2) (a) Gazieva, G. A.; Izmest'ev, A. N. Chem. Heterocycl. Compd. (N. Y., NY, U. S.) 2015, 50, 1515-1527. (b) Newberry, R. W.; VanVeller, B.; Raines, R. T. Chem. Commun. 2015, 51, 9624-9627. (c) Stadelman, B. S.; Brumaghim, J. L. ACS Symp. Ser. 2013, 1152, 33-70. (d) Bhanu, V. N.; Rao, N. V.; Lakshmi, B. M.; Harika, V.; Desu, P. K.; Nama, S. Int. J. Pharm. Chem. Res. 2012, 1, 6-14. (e) Gu, X.; Zhu, Y. Z. Expert Rev. Clin. Pharmacol. 2011, 4, 123-133. (f) Zhang, Y.; Munday, R. Mol. Cancer Ther. 2008, 7, 3470-3479.

(3) Okuma, K. Phosphorus, Sulfur Silicon Relat. Elem. 2013, 188, 340348

(4) (a) Rao, V. P. Sulfur Rep. 1992, 12, 359-403. (b) Ramnath, N.; Ramesh, V.; Ramamurthy, V. J. Org. Chem. 1983, 48, 214-222.

(5) (a) Polshettiwar, V.; Kaushik, M. P. J. Sulfur Chem. 2006, 27, 353-386. (b) Cooper, N. J. Compr. Org. Funct. Group Transform. II 2005, 3, 355-396.

(6) (a) Polshettiwar, V.; Kaushik, M. P. Tetrahedron Lett. 2004, 45, 6255-6257. (b) Curphey, T. J. J. Org. Chem. 2002, 67, 6461-6473. (c) Bottcher, B.; Bauer, F. Justus Liebigs Ann. Chem. 1951, 574, 218226.

(7) (a) Nguyen, T. T.; Le, T. N.; Hansen, P. E.; Duus, F. Tetrahedron Lett. 2006, 47, 8433-8435. (b) Elofson, R. M.; Baker, L. A.; Gadallah, F. F.; Sikstrom, R. A. J. Org. Chem. 1964, 29, 1355-1357. (c) Gofton, B. F.; Braude, E. A. Org. Synth. 1955, 35, 97-98. (d) Mayer, R.; Hiller, G.; Nitzschke, M.; Jentzsch, J. Angew. Chem. 1963, 75, 1011-1014.

(8) Pathak, U.; Pandey, L. K.; Tank, R. J. Org. Chem. 2008, 73, 28902893. 
(9) Degl'Innocenti, A.; Capperucci, A.; Mordini, A.; Reginato, G.; Ricci, A.; Cerreta, F. Tetrahedron Lett. 1993, 34, 873-876.

(10) Ray, S.; Bhaumik, A.; Dutta, A.; Butcher, R. J.; Mukhopadhyay, C. Tetrahedron Lett. 2013, 54, 2164-2170.

(11) Lecher, H. Z.; Greenwood, R. A.; Whitehouse, K. C.; Chao, T. H. J. Am. Chem. Soc. 1956, 78, 5018-5022.

(12) Wang, Z. In Comprehensive Organic Name Reactions and Reagents; John Wiley \& Sons, Inc.: New York, 2010.

(13) (a) Scheibye, S.; Pedersen, B. S.; Lawesson, S. O. Bull. Soc. Chim. Belg. 1978, 87, 229-238. (b) Pedersen, B. S.; Scheibye, S.; Nilsson, N. H.; Lawesson, S. O. Bull. Soc. Chim. Belg. 1978, 87, 223228. (c) Pedersen, B. S.; Scheibye, S.; Clausen, K.; Lawesson, S. O. Bull. Soc. Chim. Belg. 1978, 87, 293-297.

(14) Cava, M. P.; Levinson, M. I. Tetrahedron 1985, 41, 5061-5087.

(15) (a) Kayukova, L. A.; Praliyev, K. D.; Gut'yar, V. G.; Baitursynova, G. P. Russ. J. Org. Chem. 2015, 51, 148-160.

(b) Magalhaes de Moreira, D. R. Synlett 2008, 2008, 463-464.

(c) Ozturk, T.; Ertas, E.; Mert, O. Chem. Rev. 2007, 107, 5210-5278.

(d) Jesberger, M.; Davis, T. P.; Barner, L. Synthesis 2003, 1929-1958.

(16) Yokoyama, M.; Hasegawa, Y.; Hatanaka, H.; Kawazoe, Y.; Imamoto, T. Synthesis 1984, 1984, 827-829.

(17) Ori, M.; Nishio, T. Heterocycles 2000, 52, 111-116.

(18) (a) Giofre, S. V.; Romeo, R.; Mancuso, R.; Cicero, N.; Corriero, N.; Chiacchio, U.; Romeo, G.; Gabriele, B. RSC Adv. 2016, 6, 2077720780. (b) Bhowmik, S.; Mishra, A.; Batra, S. RSC Adv. 2011, 1, 1464-1470. (c) Filippi, J.-J.; Fernandez, X.; Lizzani-Cuvelier, L.; Loiseau, A.-M. Tetrahedron Lett. 2003, 44, 6647-6650.

(19) Varma, R. S.; Kumar, D. Org. Lett. 1999, 1, 697-700.

(20) Rauchfuss, T. B.; Zank, G. A. Tetrahedron Lett. 1986, 27, 34453448 .

(21) Yoshifuji, M.; An, D.-L.; Toyota, K.; Yasunami, M. Tetrahedron Lett. 1994, 35, 4379-4382.

(22) Rao, Y.; Li, X.; Nagorny, P.; Hayashida, J.; Danishefsky, S. J. Tetrahedron Lett. 2009, 50, 6684-6686.

(23) (a) Nishio, T. J. Chem. Soc., Chem. Commun. 1989, 205-206.

(b) Nishio, T. J. Org. Chem. 1997, 62, 1106-1111.

(24) Przychodzen, W. Eur. J. Org. Chem. 2005, 2005, 2002-2014.

(25) Vedejs, E.; Marth, C. F. J. Am. Chem. Soc. 1988, 110, 39483958.

(26) Davies, R.; Patel, L. In Handbook of Chalcogen Chemistry: New Perspectives in Sulfur, Selenium and Tellurium, 2nd ed.; Francesco Devillanova, W.-W. D. M., Ed.; The Royal Society of Chemistry: Cambridge, U.K., 2013; Vol. 1, pp 238-306.

(27) As mentioned above, thionation of carboxylic acids and alcohols can operate under different mechanisms, so their study, currently being investigated, is beyond the scope of this paper and will be communicated in due course.

(28) Frish, M. J.; et al. Gaussian 09, revision D1; Gaussian, Inc.: Wallingford, CT. For the full reference, see the Supporting Information

(29) Zhao, Y.; Truhlar, D. G. Acc. Chem. Res. 2008, 41, 157-167.

(30) (a) Rassolov, V. A.; Ratner, M. A.; Pople, J. A.; Redfern, P. C.; Curtiss, L. A. J. Comput. Chem. 2001, 22, 976-984. (b) Hehre, W. J.; Ditchfield, R.; Pople, J. A. J. Chem. Phys. 1972, 56, 2257-2261.

(c) Hariharan, P. C.; Pople, J. A. Theor. Chim. Acta 1973, 28, 213-222.

(31) Lam, Y.-h.; Grayson, M. N.; Holland, M. C.; Simon, A.; Houk,

K. N. Acc. Chem. Res. 2016, 49, 750-762.

(32) (a) Sumimoto, M.; Iwane, N.; Takahama, T.; Sakaki, S. J. Am. Chem. Soc. 2004, 126, 10457-10471. (b) Tamura, H.; Yamazaki, H.; Sato, H.; Sakaki, S. J. Am. Chem. Soc. 2003, 125, 16114-16126.

(33) Buncel, E.; Stairs, R. A. Solvent Effects in Chemistry, 2nd ed.; Wiley: Hoboken, NJ, 2016.

(34) Liu, C. T.; Maxwell, C. I.; Edwards, D. R.; Neverov, A. A.; Mosey, N. J.; Brown, R. S. J. Am. Chem. Soc. 2010, 132, 16599-16609.

(35) IUPAC recommends naming an encounter complex formed from two molecular entities as an encounter pair to differentiate from larger encounter complexes involving more entities. See: Muller, P. Pure Appl. Chem. 1994, 66, 1077-1184 (on page 1112).
(36) Actually, two monomers PhPSS can adopt a more stable disposition by $\sim 2.0 \mathrm{kcal} / \mathrm{mol}$ in which $\pi, \pi$-interactions are present between the two aromatic rings. However, such a disposition should come from a less stable cis dimer of LR. See the Supporting Information for a NCI study and further details.

(37) Cortes-Santiago, A.; Vazquez-Mayagoitia, A.; del Campo, J. M.; Soriano-Agueda, L. A.; Vargas, R; Garza, J. Comput. Theor. Chem. 2015, 1062, 36-43.

(38) Indeed, the use of $G_{\text {corr }}$ (see the Supporting Information for a definition) reveals such destabilization, which is erroneously ignored by the value of $G$. For the unimolecular process from LR to EP-LR, the energy values of $G$ and $G_{\text {corr }}$ are similar and fully consistent.

(39) To consider thionation starting from acetone and isolated PhPSS does not correspond to a realistic situation. Even though PhPSS is isolated, it forms aggregates like EP-LR that should be broken to form the reactive complex EP01. Disaggregation of EP-LR to form the corresponding EP01 has an energy cost that depends on the carbonyl compound.

(40) (a) Johnson, E. R.; Keinan, S.; Mori-Sanchez, P.; ContrerasGarcia, J.; Cohen, A. J.; Yang, W. J. Am. Chem. Soc. 2010, 132, 64986506. (b) Lane, J. R.; Contreras-Garcia, J.; Piquemal, J.-P.; Miller, B. J.; Kjaergaard, H. G. J. Chem. Theory Comput. 2013, 9, 3263-3266.

(41) (a) Silvi, B.; Fourre, I.; Alikhani, M. E. Monatsh. Chem. 2005, 136, 855-879. (b) Savin, A.; Becke, A. D.; Flad, J.; Nesper, R.; Preuss, H.; Vonschnering, H. G. Angew. Chem., Int. Ed. Engl. 1991, 30, 409412. (c) Savin, A.; Nesper, R.; Wengert, S.; Fassler, T. F. Angew. Chem. 1997, 36, 1808-1832.

(42) Byrne, P. A.; Gilheany, D. G. Chem. Soc. Rev. 2013, 42, 66706696.

(43) Vedejs, E.; Marth, C. F. J. Am. Chem. Soc. 1990, 112, 39053909.

(44) Beckmann, H.; Ohms, G.; Grossmann, G.; Krueger, K.; Klostermann, K.; Kaiser, V. Heteroat. Chem. 1996, 7, 111-118.

(45) (a) Abdel-Malek, H. A. Phosphorus, Sulfur Silicon Relat. Elem. 2012, 187, 506-514. (b) Verkoyen, C.; Rademacher, P. Chem. Ber. 1985, 118, 653-660. (c) Ecker, A.; Schmidt, U. Monatsh. Chem. 1972, 103, 736-743. (d) Luo, W.; Yu, Q.-s.; Tweedie, D.; Deschamps, J.; Parrish, D.; Holloway, H. W.; Li, Y.; Brossi, A.; Greig, N. H. Synthesis 2008, 2008, 3415-3422.

(46) Wen, T.; Bau, R.; McKenna, C. E. J. Chem. Soc., Chem. Commun. 1991, 1223-1224.

(47) Wang, L.; Phanstiel. J. Org. Chem. 2000, 65, 1442-1447.

(48) Baxter, S. L.; Bradshaw, J. S. J. Org. Chem. 1981, 46, 831-832.

(49) Jensen, O. E.; Lawesson, S. D.; Bardi, R.; Piazzesi, A. M.; Toniolo, C. Tetrahedron 1985, 41, 5595-5606. 